\title{
Division by multiplication
}

\author{
JAMIE I. D. CAMPBELL \\ University of Saskatchewan, Saskatoon, Saskatchewan, Canada
}

\begin{abstract}
In two experiments, item-specific transfer was examined in simple multiplication and division with prime and probe problems separated by four to six trials. As was predicted by Rickard and Bourne's (1996) identical-elements model, response time (RT) savings were larger with identical (e.g., prime $63 \div 7$, probe $63 \div 7)$ than with inverted $(63 \div 9$ and $63 \div 7)$ division problems, whereas identical $(7 \times 9$ and $7 \times 9)$ and inverted $(9 \times 7$ and $7 \times 9)$ multiplication problems produced equivalent transfer. Nonetheless, there was statistically significant transfer between inverted division problems. Furthermore, RT savings in the multiplication-to-division transfer conditions (e.g., prime $7 \times 9$, probe $63 \div 7$ ) indicated that multiplication mediated large-number division problems. These latter effects are not predicted by the identical-elements model but may be reconciled with the model by distinguishing associative transfer (facilitation owing to strengthening of a common problem node in memory) from mediated transfer (facilitation owing to mediation by a strengthened, related problem). Skilled adults can exploit the conceptual correspondences between multiplication and division facts in a highly efficient way to facilitate performance.
\end{abstract}

Over the last 20 years, research into the cognitive arithmetic skills of educated adults has focused almost exclusively on modeling the memory processes that subserve number fact retrieval (see Ashcraft, 1992, 1995, for excellent reviews). This focus was motivated by the largely unquestioned assumption that skilled adults rely more or less exclusively on direct retrieval to solve single-digit problems in the four operations (see Baroody, 1994). Recently, however, researchers increasingly have found evidence that educated adults employ a variety of procedural strategies (e.g., counting, decomposition, transformation, etc.), even for elementary problems such as $6+9$ or $7 \times 6$ (Geary \& Wiley, 1991; LeFevre, Bisanz, et al., 1996; LeFevre, Sadesky, \& Bisanz, 1996). Adults' extensive knowledge of conceptual and functional relationships among problems and operations apparently can provide a relatively efficient procedural basis for arithmetic performance.

This article examines the relation between adults' performance of simple division and multiplication problems, such as $6 \times 8=48$ and $48 \div 6=8$. The central issue is the extent to which the two operations are interdependent. One possibility is that corresponding problems in the two operations are represented and retrieved as independent facts in memory. In support of this, Rickard and Bourne (1996; Rickard, Healy, \& Bourne, 1994) found little trans-

\footnotetext{
This research was funded by a grant from the Natural Sciences and Engineering Research Council of Canada. The author thanks Mark Ashcraft, Morton Gernsbacher, Jo-Anne LeFevre, and Jane Zbrodoff for helpful comments on a previous version of this manuscript. The author also thanks Andrea Ludtke, who coordinated data collection for the present study. Correspondence concerning this article should be addressed to J. I. D. Campbell, Department of Psychology, University of Saskatchewan, 9 Campus Drive, Saskatoon S7N 5A5, Canada (e-mail: jamie.campbell(a)usask.ca).
}

fer of practice between multiplication and division problems. Specifically, they gave adults extensive practice on subsets of simple multiplication and division problems and then tested performance on the same problems (identical repetition), problems with operand order reversed (order change), corresponding problems in the other operation (operation change), and "new" unpracticed control problems. They observed transfer of practice (i.e., response time [RT] savings) in the order change condition for multiplication (e.g., $7 \times 8$ and $8 \times 7$ ) that was nearly as strong as that with identical repetition. They found no transfer between the two orders of division problems (e.g., $56 \div 7=$ 8 and $56 \div 8=7$ ), however, and little evidence that practice transferred in either direction between corresponding division and multiplication problems. If such transfer primarily measures strengthening of retrieval processes, these results imply that people's performance of corresponding division and multiplication problems did not depend on a common retrieval process. Consistent with this, Cipolotti and de Lacy Costello (1995) described an acalculic patient whose multiplication memory was largely intact, but who nonetheless presented severely impaired division performance.

On the basis of their transfer findings, Rickard et al. (1994; Rickard \& Bourne, 1996) proposed an identicalelements model of asymptotic memory for multiplication and division facts. According to the model, each memorized number fact (e.g., $6 \times 8=48$ ) is represented by a memory node that consists of the two problem operands (e.g., 6 and 8), the answer (e.g., 48), and the arithmetic operation (e.g., multiply). A central theoretical claim of the model is that problems that have the same problem operands are represented by a common node. Hence, multiplication problems that differ only in operand order, such as $6 \times 8$ and $8 \times 6$, access the same node. In contrast, problems that differ with respect to even one element ac- 
cess different nodes. Thus, inverse division problems (e.g., $48 \div 6$ and $48 \div 8$ ) are represented by different nodes. Because practice strengthens only the node corresponding to the practiced problem, transfer occurs between the two orders of multiplication problems, but there is no transfer between the two orders of division problems and no transfer between corresponding multiplication and division facts.

Although the transfer data presented by Rickard et al. (1994; Rickard \& Bourne, 1996) clearly support the identical-elements model, the cognitive relation between division and multiplication may often be more involved than the model implies. Typically, multiplication facts are learned in school before division, and children apparently rely initially on their knowledge of addition and multiplication to solve division problems (Geary, 1994; Siegler, 1988). These conditions could promote a system in which division often is mediated by multiplication. This is consistent with the impaired multiplication and division performance of a patient with brain injury, described by Hittmair-Delazer, Semenza, and Denes (1994). The 45year-old patient presented parallel patterns of loss and preservation for corresponding multiplication and division problems, and rehabilitation of multiplication transferred to division. Hittmair-Delazer et al. concluded that the patient's division performance depended on the availability of the corresponding multiplication fact.

Campbell (1997) also found evidence that adults' performance of simple division involved multiplication. In this study, university students were given division problems that alternated with multiplication problems under instructions for speeded responses. RTs were highly correlated for corresponding division and multiplication problems, and error characteristics indicated parallel retrieval structures. Specifically, division errors were constrained by semantic distance in divisor units (i.e., the distance between the presented dividend and the product implied by the error), rather than by distance from the correct quotient. This indicates that memory for division is organized in terms of multiplicative relationships and is consistent with the hypothesis that division was based on memory for multiplication facts. Analysis of intertrial error priming (see Campbell \& Clark, 1989), however, complicated the picture. By comparing errors with the answers or problems encountered during the 10 trials preceding an error, Campbell (1997) showed that multiplication errors (e.g., $7 \times 9=56$ ) were primed by previous multiplication trials $(8 \times 7=56)$ and also by previous division trials $(56 \div 7=8)$. In contrast, whereas division errors $(56 \div 7=9)$ were primed by previous division trials $(63 \div 9=7)$, division errors were not primed by previous multiplication trials.

To account for asymmetrical error priming (i.e., division to multiplication priming, but not multiplication to division priming), Campbell suggested that the performance of corresponding division and multiplication problems involved separate memory representations, as is assumed in the identical-elements model, but that par- ticipants frequently used multiplication as a check after direct retrieval of the quotient. The multiplication check on division trials would prime later multiplication errors, as was observed (i.e., division to multiplication error priming). In contrast, multiplication trials would not be expected to prime division errors, because incorrect division trials reflect errors of direct retrieval and division is not used to check multiplication.

This explanation raises an obvious question, however. If adults often use multiplication to mediate or check division, why did Rickard et al. (1994) and Rickard and Bourne (1996) not observe transfer between division and multiplication? There were at least two salient methodological differences between Campbell's (1997) errorpriming experiment and Rickard et al.'s transfer studies that could be important. First, in Campbell's (1997) study, error priming was observed over a range of only about 10 trials, whereas Rickard et al. tested transfer after longer delays (e.g., in separate test blocks at the end of practice or after a 1 day layoff from practice). The error-priming analysis may have measured short-term transfer effects that are not observed at longer delays. Second, the Rickard et al. and Rickard and Bourne studies involved many more repetitions of problems than did Campbell's (1997) study. During practice, participants received each problem 40 times in the Rickard et al. study and 90 times in the Rickard and Bourne study. Each repetition presumably would increase the probability of direct retrieval the next time the problem was encountered. Indeed, Rickard et al. (1994, p. 1149) suggested that the evidence of independent processes for division and multiplication might have partially reflected learning within their experiments. In the Campbell (1997) study, there were only three repetitions per item; consequently, that experiment potentially provided a better measure of the dependencies between division and multiplication that existed prior to the experiment.

\section{EXPERIMENT 1}

To pursue these issues, the following experiment measured transfer between division and multiplication within 72-trial blocks comprised of 36 prime-probe problem pairs. The prime-probe pairs represented identical repetition, order change, and operation change conditions for both simple division and multiplication problems. The two problems in each pair were separated by a number of intervening trials. The experiment included short primeprobe lags of 4,5 , or 6 trials and relatively long lags of 50 trials, on average. Transfer is measured by comparing probe performance with performance in the appropriate prime condition. The following paragraphs outline predictions for transfer associated with different functional relations between division and multiplication.

\section{Simple Direct Retrieval}

People may solve division by direct retrieval, with multiplication playing no role whatsoever. If we assume, 
as in the identical-elements model, that corresponding division and multiplication facts are not mediated by a common node and that multiplication is not used in any fashion on division trials, no transfer between division and multiplication in either direction is expected.

\section{Direct Division Retrieval With \\ a Postretrieval Check by Multiplication}

Although division and multiplication facts may be represented independently and usually answered by direct retrieval, people may have relatively low confidence in their division skill and use multiplication to check the result of direct division retrieval (i.e., the multiplication check occurs after the division response has been given). In the experimental context, this would insert a covert practice trial involving the corresponding multiplication problem at that point in the trial sequence. The covert retrieval potentially would strengthen the multiplication node and produce savings the next time that node was accessed. Because multiplication is typically the better learned operation, multiplication would be used to check division, but division is not likely to be used to check multiplication. Consequently, the covert transfer effect is expected to be asymmetrical: As Campbell (1997) observed for intertrial error priming, if multiplication is used only to check division, division trials should prime or produce savings for multiplication, but multiplication trials are not expected to transfer to division. No multiplication-todivision transfer would occur, because the multiplication check is assumed to occur after direct retrieval of the division fact and, therefore, multiplication would not contribute directly to division performance.

\section{Division Mediated by Multiplication}

If people sometimes use multiplication to check division, presumably it is because their division memory is relatively weak. This suggests, however, that if division memory was sufficiently weak, people would actually solve division problems by retrieving the corresponding multiplication fact (Rickard et al., 1994, p. 1149). As an example, $63 \div 7$ could be solved by using the division problem as a cue to retrieve the multiplication fact that 7 $\times 9=63$ or $9 \times 7=63$. The nonmatching operand, 9 , would then be reported as the quotient. Because division performance would depend on the accessibility of the corresponding multiplication fact, previous practice that facilitates retrieval of that multiplication fact (e.g., a recent trial involving that multiplication problem) would transfer to division. Thus, in contrast to the checking hypothesis, the mediation hypothesis predicts transfer from multiplication to division. Whether or not transfer is observed in the other direction (i.e., from division to multiplication) depends on how the multiplication fact is processed during mediation. If the covert multiplication process mediating division produces strengthening of the multiplication node, we would expect transfer from division to corresponding multiplication problems. In this case, we expect transfer in both directions, given that division is mediated by multiplication.

Rickard and Bourne (1996, p. 1284) found equivalent transfer effects for small and large problems; nonetheless, in looking for evidence of mediation, it may be important to separately examine performance for small- and largenumber problems. In general, small-number problems (e.g., $2 \times 3,6 \div 2$ ) are answered more quickly and accurately than larger problems $(6 \times 8,48 \div 6)$, the socalled problem-size effect. Small-number problems may be better learned because they are practiced more often (Ashcraft, 1995; Ashcraft \& Christy, 1995; Geary, 1996), although it is likely that other factors contribute to the effect (Campbell, 1995; LeFevre, Bisanz, et al., 1996; LeFevre, Sadesky, \& Bisanz, 1996; Zbrodoff, 1995). For example, on the basis of retrospective verbal protocols, LeFevre, Sadesky, and Bisanz (1996) proposed that small-number problems are more likely to be solved by direct retrieval than are large-number problems, which have a higher probability of solution by slower procedural strategies (e.g., transformation, decomposition). Similarly, small-number divisions may be more likely to be solved by direct retrieval, whereas large-number problems may be relatively more likely to be mediated. To examine this in the present experiment, transfer was analyzed separately for small-number and large-number problems.

\section{Method}

Participants. Thirty volunteers ( 13 females, 17 males) were recruited from the participant pool operated by the Department of Psychology or through advertisements posted around the University of Saskatchewan. The advertisement described the experiment as a study of simple arithmetic skills. The participants ranged in age from 18 to 47 years $(M=26.2$ ), reported normal or correctedto-normal vision, and were paid $\$ 6$ for their participation.

Apparatus. Stimuli appeared on two high-resolution monitors using an IBM-type personal computer, with one monitor being viewed by the experimenter and the other by the participant. Stimuli appeared horizontally as white characters against a dark background. The participant sat approximately $50 \mathrm{~cm}$ from the monitor and wore a lapel microphone that activated a relay switch connected to the computer's serial port. The sound-activated relay controlled a software clock that was accurate to $\pm 1 \mathrm{msec}$.

Stimuli and Design. Stimuli were the multiplication problems composed of pairs of arabic digits between 2 and 9 (i.e., $2 \times 2$ through $9 \times 9)$ and the corresponding division problems $(4 \div 2$ through $81 \div 9$ ). The two operands in a problem were separated by the operation sign $(\div$ or $X)$ with adjacent spaces. There are 36 possible pairings of the numbers 2 through 9 when commuted pairs (e.g., $3 \times 8$ and $8 \times 3$ ) are counted as one problem. The set of 36 includes 8 multiplication tie problems involving a single repeated operand (e.g., $2 \times 2,5 \times 5$ ) and 28 multiplication nontie problems (e.g., $2 \times 3,5 \times 7$ ). There are 8 corresponding division ties $(4 \div 2$, $25 \div 5)$ and 28 division nonties $(6 \div 2,35 \div 7)$. Digits were $7 \mathrm{~mm}$ high and $3 \mathrm{~mm}$ wide, and problems were approximately $15 \mathrm{~mm}$ in total width.

Each participant received 432 transfer tests involving prime probe problem pairs separated by some number of intervening trials. The relation between the prime and probe defined six transfer conditions: 
(1) identical multiplication prime and probe (e.g., prime $6 \times 9$ probe $6 \times 9$ ), (2) identical division prime and probe (e.g., prime 54 $\div 6$, probe $54 \div 6$ ), (3) multiplication prime and probe with operand order changed (e.g., prime $6 \times 9$, probe $9 \times 6$ ), (4) division prime and probe with order changed (e.g., prime $54 \div 9$, probe $54 \div 6$ ), (5) operation change with a division prime and multiplication probe (e.g., prime $54 \div 9$, probe $6 \times 9$ ), and (6) operation change with a multiplication prime and division probe (e.g., prime $6 \times 9$, probe $54 \div$ 6 ). Both short and long transfer distances were tested. Short transfer distances were prime-probe lags of 4,5 , or 6 trials. Long transfer distances were prime-probe lags of 50,51 , and between 30 and 70 trials. The latter, variable long-lag condition corresponds to an average lag of about 50 trials. Tie problems were included in all conditions to simplify programming but were excluded from analysis because the within-operation manipulation of operand order is irrelevant for ties.

The participants received 12 blocks of 72 problems, for a total of 864 trials. Each 72-trial block included 36 multiplication trials and 36 division trials, with 1 prime-probe pair being based on each of the 36 number pairs (e.g., $6 \times 9,9 \times 6,54 \div 6$, and $54 \div 9$ are based on the pair 6,9 ). Across the 12 blocks, each of the 36 number pairs was tested in each of the six transfer conditions, using both a short and a long transfer distance. Within a block, there were 6 prime-probe pairs in each of the six transfer conditions, and three transfer conditions were tested with short and three with long distances. In the following block, the assignment of transfer conditions to short and long lags was reversed. Thus, all 12 transfer condition $X$ lag combinations were tested six times in each pair of successive odd- and even-numbered blocks. The frequencies of the six transfer conditions in odd- and even-numbered blocks was balanced across participants, as was the assignment of the six transfer conditions to specific short lags (i.e., 4, 5, or 6 trials) and long lags (i.e., 50, 51, and between 30 to 70 trials). Over all trials, operand order (i.e., $3 \times$ 6 vs. $6 \times 3 ; 18 \div 3$ vs. $18 \div 6$ ) was random with respect to transfer condition, but across all conditions, all the problems were tested exactly six times in each order. To avoid repeating sequences of problems or answers, the sequencing program avoided more than two successive transfer tests involving the same lag. Within these constraints, the order of conditions across blocks for each problem and the order of problems in each block were independently randomized for each participant.

Procedure. The participants were tested individually with an experimenter present. Instructions described the experiment as a test of speed of simple numerical skills in which the task was to state the correct answer as quickly as possible. The participants were also advised that occasional errors are normal with speeded responding, and the experimenter occasionally reminded the participants to respond as quickly as possible. The emphasis on speed was intended to generate error rates sufficient to measure transfer in terms of error savings, in addition to RT savings.

The experimenter initiated each block of trials. Each block consisted of a continuous sequence of trials with nothing to differentiate primes and probes. For each trial, a fixation dot appeared at the center of the screen and flashed off-on-off over a 1 -sec interval. The problem appeared on what would have been the next flash with the operation sign $(\div$ or $\times)$ at fixation. Timing began when the stimulus appeared and ended when the response triggered the sound-activated relay. When a sound was detected, the displayed problem was replaced instantly by the fixation dot. This allowed the experimenter to detect premature or delayed triggering of the relay and to mark such trials as spoiled. The l-sec fixation dot began to flash as soon as the experimenter entered the subject's answer; thus, the response-stimulus interval was usually slightly longer than $1 \mathrm{sec}$. No feedback was provided regarding either speed or accuracy. The participants were offered a brief rest period between blocks and were reminded about the importance of speed. Prior to the arithmetic task, the participants named as quickly as possible the Arabic numbers 1 through 40, presented individually in a random order. This provided an opportunity to adjust the sensitivity of the sound-activated relay. Testing required about $1 \mathrm{~h}$.

\section{Results and Discussion}

The overall mean RT for correct trials in Experiment 1 was $912 \mathrm{msec}$, and the overall error rate was $11.9 \%$. Separate four-way repeated measures analyses of variance (ANOVAs) of transfer in the short- and long-lag conditions were performed. The factors included problem type (prime or probe), transfer condition (identical repetition, order change, or operation change), operation (division or multiplication), and problem size (small or large). Problems with a dividend or product greater than 25 were defined as large (Campbell, 1997). This split creates two sets, each containing 14 nontie problems when commuted pairs (e.g., $4 \times 8$ and $8 \times 4$ or $32 \div 4$ and $32 \div 8$ ) are counted as one problem. A total of $155 \mathrm{RTs}$ were spoiled by failures of the voice-activated relay $(0.8 \%)$, and another $289(1.4 \%)$ were discarded as outliers more than three $S D$ s from each participant's grand mean for each operation $X$ size combination. Pairwise deletion of missing RT data (i.e., owing to errors, spoiled trials, or outliers) ensured for each participant that the mean prime RT and mean probe RT in each operation $\times$ problem size $\times$ transfer cell were based on the same set of problems (a mean of 11.7 problems out of a maximum of 14 over all conditions). Matched deletion of missing RTs could not be extended to include the operation or transfer condition factors, because it resulted in a prohibitive depletion of observations; consequently, different operation $\times$ transfer cells were not necessarily based on identical problem sets. For tests of operation change transfer, the yoked prime and probe problems involved different operations. Therefore, to obtain a baseline for operation change, each operation change probe trial was matched with the corresponding operation change prime trial from the other operation.

In all the following analyses, small problems produced significantly faster RTs and fewer errors than did large problems, as was expected. Similarly, multiplication always was significantly faster and less error prone than division, replicating previous research (e.g., Campbell, 1997; Rickard et al., 1994). Overall, small and large problems produced RT means of 855 and $969 \mathrm{msec}$, respectively, and multiplication and division produced means of 865 and $958 \mathrm{msec}$. Error rates for small and large problems were $7.6 \%$ and $16.1 \%$; for multiplication and division, they were $8.1 \%$ and $15.6 \%$. Significance tests for main effects of problem size and operation are omitted throughout. All other effects were significant at $p<.001$, unless otherwise indicated, and only effects with $p<.05$ are reported.

Short-lag probes versus short-lag primes: Response times. Table 1 presents mean RT for correct responses in the short-lag conditions as a function of operation, problem size, problem type (prime or probe), and transfer condition. The table also shows mean savings (i.e., prime RT - probe RT) and the standard error of mean 
Table 1

\begin{tabular}{|c|c|c|c|c|c|c|c|c|c|c|c|c|}
\hline \multirow[b]{3}{*}{ Problem Type } & \multicolumn{6}{|c|}{ Division Probes } & \multicolumn{6}{|c|}{ Multiplication Probes } \\
\hline & \multicolumn{2}{|c|}{$\begin{array}{l}\text { Identical } \\
\text { Repetition }\end{array}$} & \multicolumn{2}{|c|}{$\begin{array}{c}\text { Order } \\
\text { Change }\end{array}$} & \multicolumn{2}{|c|}{$\begin{array}{c}\text { Operation } \\
\text { Change }\end{array}$} & \multicolumn{2}{|c|}{$\begin{array}{l}\text { Identical } \\
\text { Repetition }\end{array}$} & \multicolumn{2}{|c|}{$\begin{array}{l}\text { Order } \\
\text { Change }\end{array}$} & \multicolumn{2}{|c|}{$\begin{array}{c}\text { Operation } \\
\text { Change }\end{array}$} \\
\hline & $M$ & $S E M$ & $M$ & $S E M$ & $M$ & $S E M$ & $M$ & $S E M$ & $M$ & $S E M$ & $M$ & $S E M$ \\
\hline \multicolumn{13}{|c|}{ Small Problems } \\
\hline Short-lag prime & 922 & & 945 & & 906 & & 815 & & 816 & & 828 & \\
\hline Short-lag probe & 840 & & 907 & & 925 & & 773 & & 770 & & 807 & \\
\hline Savings & +82 & 14.7 & +38 & 21.2 & -19 & 17.1 & +42 & 12.2 & +46 & 13.4 & +21 & 18.6 \\
\hline \multicolumn{13}{|c|}{ Large Problems } \\
\hline Short-lag prime & 1,029 & & 1,049 & & 1,062 & & 960 & & 965 & & 936 & \\
\hline Short-lag probe & 914 & & 992 & & 1,002 & & 887 & & 885 & & 941 & \\
\hline Savings & +115 & 22.1 & +57 & 32.3 & +60 & 28.7 & +73 & 17.2 & +80 & 18.7 & -5 & 20.2 \\
\hline
\end{tabular}

savings. The average RT savings on short-lag probes, relative to primes, was $49 \mathrm{msec}$ [ $887 \mathrm{vs.} 936 \mathrm{msec} ; F(1,29)=$ $\left.49.85, M S_{\mathrm{e}}=8,745\right]$. Savings, however, varied across transfer condition $\left[F(2,58)=11.50, M S_{\mathrm{e}}=62,306\right]$, with mean savings of 78,55 , and $14 \mathrm{msec}$ for identical repetition, order change, and operation change conditions, respectively. These effects were qualified, however, by a significant four-way interaction $\left[F(2,58)=3.18, M S_{\mathrm{e}}=\right.$ $5,143, p=.049]$. The four-way effect was decomposed by performing separate operation $\times$ size $\times$ problem type ANOVAs at each level of the transfer factor (i.e., identical repetition, order change, operation change). Only significant main or interaction effects involving problem type (i.e., savings) are reported.

The analysis of identical repetition indicated significant size $\times$ type $\left[F(1,29)=4.45, M S_{\mathrm{e}}=3,325, p=.044\right]$ and operation $\times$ type $\left[F(1,29)=7.70, M S_{\mathrm{e}}=3,300, p=\right.$ $.010]$ interactions. Specifically, savings from identical repetition were greater for large $(94 \mathrm{msec})$ than for small problems $(62 \mathrm{msec})$ and greater for division $(98 \mathrm{msec})$ than for multiplication $(57 \mathrm{msec})$. The large-number problems and division problems were associated with relatively long RTs and, therefore, may simply offer more room for speed-up.

In the order change analysis, the average savings of $55 \mathrm{msec}$ was reliable $\left[F(1,29)=24.16, M S_{\mathrm{e}}=7,623\right]$, and there were no significant interactions involving problem size or problem type (smallest $\alpha=.19$ ). The failure to observe an interaction between operation and problem type is potentially at odds with the identical-elements model, which predicts transfer with order change for multiplication, but not for division. Savings with an order change were significant both for division [ $48 \mathrm{msec} ; t(29)=2.17$, $p=.039$ ] and for multiplication [ $63 \mathrm{msec} ; t(29)=5.23$ ]. Nonetheless, for division, transfer with order-change $(48 \mathrm{msec})$ appeared to be substantially smaller, relative to identical repetition $(98 \mathrm{msec})$, whereas transfer with order change in multiplication $(63 \mathrm{msec})$ was essentially equivalent to identical repetition $(57 \mathrm{msec})$. The test of the corresponding interaction, however, did not reach conventional significance levels $\left[F(1,29)=2.72, M S_{\mathrm{e}}=\right.$ $8,774, p=.11]$. Thus, in contrast to the predictions of the identical-elements model, there was not clear statistical evidence that transfer with order change differed for division and multiplication.

The analysis of operation change transfer demonstrated a three-way interaction of operation, problem type, and problem size $\left[F(1,29)=6.33, M S_{\mathrm{e}}=6,687, p=.018\right]$. Thus, the four-way interaction in the overall analysis occurred mainly because there was a three-way interaction in the operation change condition but not in the identicalrepetition or order change conditions. As is shown in Table 1, the three-way interaction occurred because interoperation transfer was obtained only for the large division problems [mean savings of $60 \mathrm{msec} ; t(29)=2.09$, $p=.044]$. The average savings in the other three size $\times$ operation combinations was $-1 \mathrm{msec}$, and none of the combinations approached significance (smallest $\alpha=.26$ ). The evidence of transfer from multiplication to division is not expected according to the identical-elements model and is consistent with the hypothesis that large division problems were mediated by multiplication in the shortlag transfer condition.

Short-lag probes versus short-lag primes: Percentage of errors. Table 2 presents the mean percentage of errors in each cell of the four-factor analysis. There were significantly fewer errors on probe $(10.9 \%)$ than on prime $(12.8 \%)$ problems, which confirmed savings on errors $\left[F(1,29)=10.20, M S_{\mathrm{e}}=67.93, p=.003\right]$, but there were no significant interactions involving problem type (smallest $\alpha=.14$ ). Nonetheless, an examination of Table 2 indicates that error savings for division were due mainly to large problems with identical repetition, whereas both small and large multiplication problems tended to produce fewer errors, given identical repetition, as did large multiplication problems with order change. The 12 error savings means in Table 2 and the 12 RT savings means in Table 1 were correlated $[.53 ; p=.035$, one-tailed]. Thus, 
the pattern of error savings generally corresponded to the pattern of RT savings, although RT provided a more sensitive measure of savings.

Long-lag conditions. Mean RTs for the long-lag probes were significantly slower $(917 \mathrm{msec})$, relative to their yoked primes $\left[901 \mathrm{msec} ; F(1,29)=13.91, M S_{\mathrm{e}}=\right.$ $3,201]$. The apparent transfer costs in the long-lag RT data indicate overall faster RTs at the beginning of blocks (i.e., where long-lag primes were most common), relative to the end of blocks (i.e., where long-lag probes were most common). This might occur as a result of the experimenter's reminding the participants between blocks to respond as quickly as possible. Fatigue and accumulating interference from preceding trials may also tend to slow performance toward the end of each block. Regardless of the cause, the generally slower RTs for the long-lag probes, relative to the long-lag primes, compromises interpretation of the long-lag data with respect to item-specific transfer. Because the short-lag primes and probes were distributed more evenly throughout each trial block and were separated only by four, five, or six trials, it is very unlikely that the short-lag primes and probes involved systematically different speed-accuracy criteria.

Discussion of Experiment 1. The results of the shortlag conditions were quite straightforward. Large-number multiplication problems produced savings on corresponding division problems, but there was no transfer in the reverse direction. This pattern is consistent with multiplication mediating large-number division. There also was evidence of transfer between the two orders of division problems. Neither of these effects is predicted by the identical-elements model, but they do not necessarily contradict the model. Although the model only predicts transfer from the strengthening of a common memory node, transfer would also be observed if performance on a given problem was mediated by retrieving a related problem and if recent practice has improved the accessibility of the mediating fact. As is discussed in more detail in the General Discussion section, such a mediation process may underlie both the multiplication-to-division transfer and the cross-order division-to-division transfer observed.

Of course, we would expect especially strong transfer when the prime and the probe involve a common node. Consequently, even allowing for mediated transfer, the identical-elements model predicts stronger transfer with identical repetition than with an order change for division (i.e. because the two orders of division problems are assumed to involve separate nodes), whereas multiplicationto-multiplication transfer should be approximately equivalent for identical-repetition and order change (i.e., because commuted pairs of multiplication problems are assumed to access a common node). Although the data appeared to present this pattern, the test of the corresponding interaction was not statistically significant. One purpose of Experiment 2 was to replicate the evidence of transfer be- tween the two orders of division problems and to pursue the operation $\times$ order change interaction predicted by the identical-elements model. Experiment 2 also provided an opportunity to replicate the finding of asymmetrical interoperation transfer (i.e., from multiplication to division, but not from division to multiplication).

In Experiment 2, an attempt was also made to redress the baseline complications arising from long-lag primes occurring near the beginning and their yoked primes occurring toward the end of each block. For Experiment 2, prime-probe distances in the long-lag conditions were reduced to an average lag of 30 trials, as compared with the average of 50 trials in Experiment 1. This might reduce the chances of different speed-accuracy criteria's affecting the long-lag primes and probes.

Finally, multiplication RTs in Experiment 1 were quite fast, as compared with division RTs. If multiplication RTs were closer to their asymptote (i.e., their RT floor), it might have been more difficult to measure small transfer effects from division to multiplication RTs. This could contribute to the asymmetrical interoperation transfer (i.e., multiplication-to-division only). For Experiment 2, the experimenter reduced the emphasis on overall speed, relative to Experiment 1 , by monitoring error rates and cautioning the participants if error rates substantially exceeded $10 \%$. Reduced speed pressure ought to increase the sensitivity of the procedure for measuring RT transfer in both multiplication and division.

\section{EXPERIMENT 2}

\section{Method}

Thirty volunteers ( 17 males, 13 females) were recruited in the same way as that in Experiment 1 and attended a single session lasting about $1 \mathrm{~h}$. The participants ranged in age from 17 to 29 years $(M=21.9)$, reported normal or corrected-to-normal vision, and were paid \$6 for their participation. Except for reducing the longlag distances between primes and probes, the design of Experiment 2 was identical to that of Experiment 1 . In this experiment, long-lag distances were trial lags of 29,31 , or somewhere between 25 and 35 trials (i.e., an average of 30 ), as compared with an average of 50 trials in Experiment 1. As in Experiment 1, short transfer distances were prime-probe lags of 4,5 , or 6 trials. The mean trial numbers for long-lag primes, long-lag probes, short-lag primes, and short-lag probes were, respectively, 20.4, 50.5,33, and 38. Out of sight of the participant, the experimenter's video monitor displayed a running total of errors per block. Initial instructions emphasized speed, as in Experiment 1, but the experimenter did not intervene to comment about speed unless the participant's error rate in a block deviated substantially above or below $10 \%$.

\section{Results and Discussion}

The data from Experiment 2 were analyzed in the same way as those in Experiment 1. A total of 324 RTs were spoiled by failures of the voice-activated relay $(1.6 \%)$, and another $314(1.6 \%)$ were discarded as outliers more than three $S D$ s from the grand mean for each operation $\times$ problem size condition. The mean number of items con- 
Table 2

Mean Errors (\%) in the Short-Lag Conditions of Experiment 1

\begin{tabular}{|c|c|c|c|c|c|c|c|c|c|c|c|c|}
\hline \multirow[b]{3}{*}{ Problem Type } & \multicolumn{6}{|c|}{ Division Probes } & \multicolumn{6}{|c|}{ Multiplication Probes } \\
\hline & \multicolumn{2}{|c|}{$\begin{array}{l}\text { Identical } \\
\text { Repetition }\end{array}$} & \multicolumn{2}{|c|}{$\begin{array}{c}\text { Order } \\
\text { Change }\end{array}$} & \multicolumn{2}{|c|}{$\begin{array}{l}\text { Operation } \\
\text { Change }\end{array}$} & \multicolumn{2}{|c|}{$\begin{array}{l}\text { Identical } \\
\text { Repetition }\end{array}$} & \multicolumn{2}{|c|}{$\begin{array}{c}\text { Order } \\
\text { Change }\end{array}$} & \multicolumn{2}{|c|}{$\begin{array}{c}\text { Operation } \\
\text { Change }\end{array}$} \\
\hline & $M$ & SEM & $M$ & $S E M$ & $M$ & $S E M$ & $M$ & $S E M$ & $M$ & $S E M$ & $M$ & $S E M$ \\
\hline \multicolumn{13}{|c|}{ Small Problems } \\
\hline Short-lag prime & 11.4 & & 15.0 & & 13.3 & & 4.3 & & 2.1 & & 3.1 & \\
\hline Short-lag probe & 10.2 & & 13.8 & & 13.3 & & 0.9 & & 2.1 & & 2.1 & \\
\hline Savings & +1.2 & 1.8 & +1.2 & 1.7 & 0.0 & 2.2 & +3.3 & 0.9 & 0.0 & 0.9 & +1.0 & 1.1 \\
\hline \multicolumn{13}{|c|}{ Large Problems } \\
\hline Short-lag prime & 18.8 & & 20.2 & & 19.3 & & 15.2 & & 16.4 & & 15.0 & \\
\hline Short-lag probe & 13.6 & & 21.2 & & 17.1 & & 11.4 & & 12.1 & & 12.6 & \\
\hline Savings & +5.2 & 2.1 & -1.0 & 2.3 & +2.2 & 2.8 & +3.8 & 2.1 & +4.3 & 2.0 & +2.4 & 1.9 \\
\hline
\end{tabular}

tributing to each RT mean was 12 problems out of a maximum of 14 over all conditions. The overall mean RT for correct trials in Experiment 2 was $1,024 \mathrm{msec}$, and the error rate was $9.8 \%$. Relative to Experiment 1 , the overall mean RT in Experiment 2 was $112 \mathrm{msec}$ longer (12\%), and the overall error rate was reduced by about $20 \%$. Overall, in Experiment 2, small and large problems produced RT means of 941 and 1,106 msec, respectively, and multiplication and division produced means of 980 and $1,067 \mathrm{msec}$. Error rates for small and large problems were $5.4 \%$ and $14.2 \%$, and for multiplication and division, error rates were $8.4 \%$ and $11.2 \%$, respectively.

As in Experiment 1, the comparison of long-lag prime and probe RTs in Experiment 2 showed costs (i.e., prime RTs were faster then probe RTs). Thus, despite moving the long-lag primes and probes closer together, the tendency for the participants to respond more quickly early in blocks than later in blocks apparently still contaminated the long-lag data. Because of this, there will be no further discussion of the long-lag transfer conditions.

Short-lag probes versus short-lag primes: Response times. Table 3 presents mean RT in the short-lag conditions as a function of operation, problem size, problem type (i.e., primes vs. probes), and transfer condition. The average RT savings on short-lag probes, relative to primes, was $59 \mathrm{msec}$ [ 994 vs. $1053 \mathrm{msec} ; F(1,29)=63.62$, $\left.M S_{\mathrm{e}}=9,749\right]$. As in Experiment 1, short-lag RT savings were greater for large problems $(84 \mathrm{msec})$ than for small problems [ $\left.33 \mathrm{msec} ; F(1,29)=15.37, M S_{\mathrm{e}}=7,713\right]$. Savings also varied as a function of transfer condition, as is indicated by the significant transfer $\times$ problem type interaction $\left[F(1,29)=17.99, M S_{\mathrm{e}}=5,937\right]$. Specifically, the mean savings with identical repetition were $101 \mathrm{msec}$, with order change it was $59 \mathrm{msec}$, and with operation change it was $17 \mathrm{msec}$. There were no other significant effects (all $F \mathrm{~s}<1$ ). To decompose the transfer $\times$ problem type interaction and to present analyses that parallel those presented for Experiment 1, separate operation $\times$ problem size $\times$ problem type ANOVAs were performed at each level of the transfer factor (i.e., identical repetition, order change, and operation change). Only significant effects involving problem type (i.e., savings) are reported.

Analysis of the identical-repetition conditions confirmed a main effect of problem type [mean savings of $\left.101 \mathrm{msec} ; F(1,29)=129.07, M S_{\mathrm{e}}=4,723\right]$. Savings from identical repetition were greater for large $(136 \mathrm{msec})$ than for small problems [66 msec; $F(1,29)=11.15, M S_{\mathrm{e}}=$ $6,539, p=.002$ ], but unlike Experiment 1, there was no evidence that identical repetition benefited division more than multiplication $(F<1)$. The reduced speed pressure

Table 3

Mean Correct Response Times (in Milliseconds)

in the Short-Lag Conditions of Experiment 2

\begin{tabular}{|c|c|c|c|c|c|c|c|c|c|c|c|c|}
\hline \multirow[b]{3}{*}{ Problem Type } & \multicolumn{6}{|c|}{ Division Probes } & \multicolumn{6}{|c|}{ Multiplication Probes } \\
\hline & \multicolumn{2}{|c|}{$\begin{array}{l}\text { Identical } \\
\text { Repetition }\end{array}$} & \multicolumn{2}{|c|}{$\begin{array}{c}\text { Order } \\
\text { Change }\end{array}$} & \multicolumn{2}{|c|}{$\begin{array}{c}\text { Operation } \\
\text { Change }\end{array}$} & \multicolumn{2}{|c|}{$\begin{array}{c}\text { Identical } \\
\text { Repetition }\end{array}$} & \multicolumn{2}{|c|}{$\begin{array}{c}\text { Order } \\
\text { Change }\end{array}$} & \multicolumn{2}{|c|}{$\begin{array}{c}\text { Operation } \\
\text { Change }\end{array}$} \\
\hline & $M$ & $S E M$ & $M$ & $S E M$ & $M$ & $S E M$ & $M$ & $S E M$ & $M$ & $S E M$ & $M$ & $S E M$ \\
\hline \multicolumn{13}{|c|}{ Small Problems } \\
\hline Short-lag prime & 1,010 & & 1,011 & & 990 & & 906 & & 921 & & 907 & \\
\hline Short-lag probe & 926 & & 986 & & 1,009 & & 858 & & 863 & & 906 & \\
\hline Savings & +84 & 18.0 & +25 & 15.4 & -19 & 25.0 & +48 & 17.7 & +58 & 15.8 & +1 & 14.5 \\
\hline \multicolumn{13}{|c|}{ Large Problems } \\
\hline Short-lag prime & 1,215 & & 1,193 & & 1,164 & & 1,119 & & 1,110 & & 1,089 & \\
\hline Short-lag probe & 1,073 & & 1,120 & & 1,107 & & 990 & & 1,032 & & 1,063 & \\
\hline Savings & +142 & 22.2 & +73 & 29.0 & +57 & 25.0 & +129 & 21.5 & +78 & 27.4 & +26 & 25.1 \\
\hline
\end{tabular}


Table 4

Mean Errors (\%) in the Short-Lag Conditions of Experiment 2

\begin{tabular}{|c|c|c|c|c|c|c|c|c|c|c|c|c|}
\hline \multirow[b]{3}{*}{ Problem Type } & \multicolumn{6}{|c|}{ Division Probes } & \multicolumn{6}{|c|}{ Multiplication Probes } \\
\hline & \multicolumn{2}{|c|}{$\begin{array}{l}\text { Identical } \\
\text { Repetition }\end{array}$} & \multicolumn{2}{|c|}{$\begin{array}{l}\text { Order } \\
\text { Change }\end{array}$} & \multicolumn{2}{|c|}{$\begin{array}{l}\text { Operation } \\
\text { Change }\end{array}$} & \multicolumn{2}{|c|}{$\begin{array}{l}\text { Identical } \\
\text { Repetition }\end{array}$} & \multicolumn{2}{|c|}{$\begin{array}{l}\text { Order } \\
\text { Change }\end{array}$} & \multicolumn{2}{|c|}{$\begin{array}{c}\text { Operation } \\
\text { Change }\end{array}$} \\
\hline & $M$ & SEM & $M$ & SEM & $M$ & $S E M$ & $M$ & SEM & $M$ & $S E M$ & $M$ & $\overline{S E M}$ \\
\hline \multicolumn{13}{|c|}{ Small Problems } \\
\hline Short-lag prime & 8.8 & & 9.3 & & 10.2 & & 2.9 & & 2.4 & & 2.1 & \\
\hline Short-lag probe & 5.5 & & 8.1 & & 9.0 & & 1.9 & & 2.1 & & 2.9 & \\
\hline Savings & +3.3 & 1.7 & +1.2 & 1.9 & +1.2 & 2.0 & +1.0 & 0.9 & 0.3 & 0.9 & -0.7 & 1.3 \\
\hline \multicolumn{13}{|c|}{ Large Problems } \\
\hline Short-lag prime & 15.9 & & 15.7 & & 12.9 & & 16.7 & & 18.3 & & 15.7 & \\
\hline Short-lag probe & 9.8 & & 15.0 & & 14.3 & & 11.4 & & 11.2 & & 13.3 & \\
\hline Savings & +6.2 & 1.9 & +0.7 & 2.6 & -1.4 & 2.2 & +5.3 & 1.6 & +7.1 & 1.3 & +2.4 & 2.6 \\
\hline
\end{tabular}

(and generally longer RTs) in Experiment 2 than in Experiment 1 may have provided more equal opportunities for the two operations to demonstrate RT savings.

In the order change condition, the average savings of $59 \mathrm{msec}$ was significant $\left[F(1,29)=18.27, M S_{\mathrm{e}}=11,395\right]$, and, as in Experiment 1, there were no significant interactions involving problem type or operation (smallest $\alpha=.16)$. RT savings with an order change occurred both for division [49 msec, $t(29)=2.80, p=.009$ ] and for multiplication [68 msec, $t(29)=3.91, p=.001]$. Thus, Experiment 2 confirmed transfer between the two orders of division problems over relatively short lags, an effect not predicted by the identical-elements model. The identical-elements model also predicts stronger transfer for identical repetition than for order change for division, but not for multiplication. An ANOVA that included both identical-repetition and order change conditions (but excluded operation change) did not confirm the expected operation $\times$ problem type $\times$ transfer interaction $\left[F(1,29)=1.78, M S_{\mathrm{e}}=8,210, p=.19\right]$.

The operation change analysis indicated a two-way interaction between problem type and problem size $\left[F(1,29)=6.55, M S_{\mathrm{e}}=5,908, p=.016\right]$. As Table 3 shows, this represents larger savings for large problems $(42 \mathrm{msec})$ than for small problems $(-9 \mathrm{msec})$. The threeway interaction among operation, problem size, and problem type was not significant $(F<1)$. Nonetheless, Table 3 indicates that, as in Experiment 1, only the large division problems showed significant savings with an operation change [ $57 \mathrm{msec} ; t(29)=2.28, p=.015$, onetailed]. The average savings in the other three size $\times$ operation combinations were $4 \mathrm{msec}$, and none approached significance (smallest $\alpha=.31$ ). Thus, Experiment 2 replicated both the evidence of multiplication-to-division transfer and the absence of division-to-multiplication transfer found in Experiment 1.

Short-lag probes versus short-lag primes: Percentage of errors. Table 4 presents the mean percentage of errors in each condition. Probe trials produced fewer errors than prime trials, overall $[8.7 \%$ vs. $10.9 \% ; F(1,29)=$ 13.26, $\left.M S_{\mathrm{e}}=65.75\right]$, but problem type interacted with other variables. Specifically, error savings were greater for large than for small problems [mean savings of 3.4\% vs. $\left.1.0 \% ; F(1,29)=5.39, M S_{\mathrm{e}}=45.75, p=.027\right]$, but as Table 4 shows, this effect arose mainly from multiplication trials $\left[F(1,58)=9.72, M S_{\mathrm{e}}=27.15, p=.004\right]$. Error savings also varied as a function of transfer condition: For identical repetition, order change, and operation change, respectively, the mean error savings were $3.9 \%, 2.3 \%$, and $0.3 \%\left[F(2,58)=4.00, M S_{\mathrm{e}}=48.04, p=.024\right]$. Table 4 shows that error savings for division occurred for both small and large problems with identical repetition, whereas there was no evidence of error savings in other division conditions. For multiplication, error savings were observed for large problems, both with identical-repetition and order change primes. The 12 error savings means in Table 4 and the 12 RT savings means in Table 3 were correlated $[.69 ; p=.006$, one-tailed]. Thus, as in the shortlag conditions of Experiment 1, the pattern of error savings generally corresponded to the pattern of RT savings, although, again, RTs apparently provided a more sensitive measure.

\section{EXPERIMENTS 1 AND 2 COMBINED}

The short-lag conditions in the two experiments were practically identical; consequently, the two data sets were combined into a single set, to provide a maximally sensitive analysis of two theoretically important issues: transfer with order change and transfer with operation change. As the pattern of error savings parallelled the pattern of RT savings in both experiments, the combined analysis was restricted to the RT data, which appeared to provide a more sensitive measure of transfer. Figure 1 presents mean RT savings as a function of operation, problem size, and transfer condition for the 60 participants in Experiments 1 and 2 . The error bars represent one standard error of the mean.

The combined analysis clearly demonstrated transfer across the two orders of division problems $[F(1,59)=$ $12.02, M S_{\mathrm{e}}=11,734$ ], both for small [32 msec, $t(59)=$ $2.48, p=.016]$ and for large problems [ $65 \mathrm{msec} ; t(59)=$ $3.00, p=.004]$. The critical effect for the identicalelements model, however, concerns the prediction that 

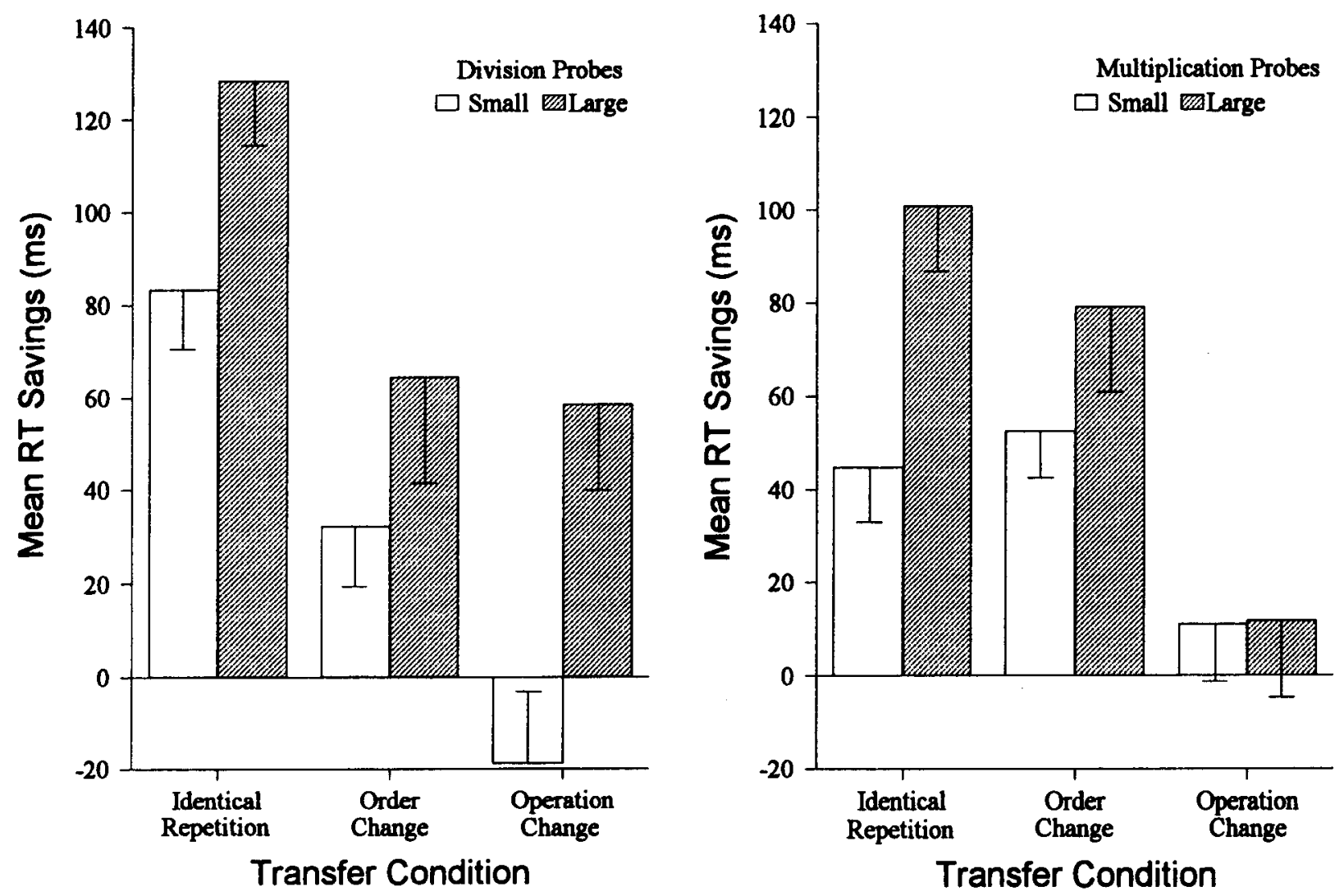

Figure 1. Mean short-lag response time savings as a function of operation, problem size, and transfer condition (Experiments 1 and 2 combined).

transfer should be stronger with identical repetition than with order change for division, but not for multiplication. Using the combined data, an ANOVA that included both identical-repetition and order change conditions (but excluded operation change) confirmed the expected operation $\times$ problem type $\times$ transfer interaction $[F(1,59)=$ 4.53, $\left.M S_{\mathrm{e}}=5,370, p=.037\right]$. Specifically, transfer in division was smaller with order change $(48 \mathrm{msec})$ than with identical repetition ( $106 \mathrm{msec}$ ), whereas RT savings in multiplication were essentially the same with order change (66 msec) or identical repetition $(73 \mathrm{msec})$.

With respect to operation change transfer, the combined analysis confirmed the three-way interaction of operation, problem size, and problem type $\left[F(1,59)=4.73, M S_{\mathrm{e}}=\right.$ $9,671, p=.034]$. As Figure 1 shows, the interaction occurred because the only significant interoperation savings involved transfer from multiplication to division for large problems [58 msec; $t(59)=3.11, p=.003] .{ }^{1}$ This effect suggests that large-number division problems were mediated by multiplication in the operation change condition. Whereas division RTs were generally longer than multiplication RTs, this difference was smaller for large $(53 \mathrm{msec})$ than for small problems $[110 \mathrm{msec} ; F(1,59)=$ 5.72, $M S_{\mathrm{e}}=8,722, p=.020$; see Tables 1 and 3]. This suggests that mediated division for large problems was quite efficient, inasmuch as the operation effect was smaller for the problems that apparently were more likely to be mediated.

\section{GENERAL DISCUSSION}

The General Discussion is divided into four sections. The first section discusses the evidence that large-number division problems were mediated by multiplication. The second section considers explanations for asymmetrical transfer - that is, the finding of transfer from multiplication to division, but not from division to multiplication. The third section addresses the use of multiplication to check rather than to mediate division, and the final section discusses implications of the results for the identicalelements model (Rickard \& Bourne, 1996).

\section{Mediation of Division by Multiplication}

The short-lag conditions of Experiments 1 and 2 showed that large-number division problems (e.g., $63 \div$ $7=$ ?) benefited from retrieval of the corresponding multiplication fact (e.g., $7 \times 9=63$ ) during the preceding four to six trials. This effect is expected if multiplication mediated division, because division performance ought to improve with the accessibility of the mediating multi- 
plication fact. It is unlikely that the multiplication-todivision transfer could be due to a simple priming mechanism, given the combined effects of interference from several intervening problems and the approximately 15 sec elapsed time, on average, between the prime and the probe. Furthermore, if the observed transfer reflected a simple priming process, we would also expect transfer from multiplication to small division problems and from division to multiplication, but these effects were not observed.

Experiments 1 and 2 apparently demonstrated mediation of division by multiplication, but to what extent can the results be generalized beyond the conditions of these experiments? In the short-lag conditions, episodic or semantic information associated with the recent prime trial might be easily reactivated by the probe. Mediating facts would not necessarily be as accessible under different conditions. Nonetheless, the experiment did not promote mediation in haphazard or improbable ways. For example, the small-number problems in the basic operations are usually well memorized and, therefore, would be more likely to be solved by direct retrieval, rather than by mediation (LeFevre, Bisanz, et al., 1996; LeFevre, Sadesky, \& Bisanz, 1996). Consistent with this, interoperation transfer occurred for large-number division problems, but there was no evidence of multiplication-to-division transfer for small-number division problems. This cannot be attributed to a general difficulty in measuring transfer for the small division problems, because these items demonstrated substantial transfer in the identical-repetition condition. Rather, the multiplication-to-division transfer results suggest that large division problems were more likely to be mediated by multiplication than were small division problems.

What might be the nature of the mediation process? Mediation implies knowledge that multiplication and division are inverse operations and, more specifically, that there is a logical correspondence between the numerical components of division and multiplication facts. For example, the division problem $63 \div 7$ would serve as a retrieval cue for the multiplication fact that $7 \times 9=63$ or $9 \times 7=63$. The number that is present in the multiplication fact and not present in the division stimulus (i.e., 9) must be the quotient. In this view, mediation entails a match-and-select process that identifies the matching numbers in corresponding division stimuli and multiplication facts and selects the nonmatching component of the multiplication fact as the quotient.

Although it is often assumed that strategies involving transformations or mediation would be inefficient, relative to direct retrieval, the present results suggest that division mediated by multiplication may be very efficient. Although larger division problems apparently were mediated by multiplication more than were smaller division problems, the mean RT difference between small division and multiplication problems was actually larger than the difference between large division and multiplication problems. Thus, mediation did not lead to greater RT costs for large division problems, relative to small division problems. More generally, mediation occurred despite a moderately high level of speed pressure, which ought to reduce the opportunity for mediation and encourage people to rely on direct retrieval. Furthermore, the multiplicationto-division mediation process was sufficiently robust to be detected (as RT savings), despite six repetitions of each problem in each operand order. Although repetition of an individual item presumably will lead to direct retrieval eventually, the present data suggest that mediation can be efficient enough to contribute to performance even after several recent repetitions of the probe item. The capacity to incorporate mediation under these conditions suggests that skilled adults can exploit the conceptual correspondence between multiplication and division in a highly efficient way to facilitate performance.

\section{Asymmetrical Interoperation Transfer}

Whereas there was clear evidence of multiplicationto-division transfer for large division problems, there was no evidence of transfer in the opposite direction (i.e., from division to multiplication). This asymmetry is rather surprising: If division were mediated consistently by retrieval of the corresponding multiplication fact, we might expect the multiplication node to be strengthened and to produce transfer from division primes to corresponding multiplication probes. The failure to observe division-tomultiplication transfer cannot reflect a general difficulty in measuring transfer to multiplication in these experiments, because there was robust transfer for multiplication problems in the within-operation transfer conditions.

Two explanations for the asymmetrical transfer suggest themselves. First, mediation of division by multiplication may depend on the accessibility of the mediating multiplication fact. For example, it is possible that the division probes were mediated by multiplication in the multiplication-to-division condition because the corresponding multiplication primes were recently retrieved (i.e., in the preceding four to six trials) and were, therefore, highly accessible. In contrast, division primes may have been less likely to be solved by mediation, because they were not preceded recently by the corresponding multiplication problem. In this case, we would not observe transfer from division to multiplication, because division primes in the division-to-multiplication transfer condition were relatively unlikely to be mediated by multiplication. Alternatively, the asymmetrical transfer (i.e., multiplication-to-division but not division-to-multiplication) could indicate that the process by which multiplication mediated division did not produce strong priming or strengthening of the multiplication node. For example, there may be no intentional review or consolidation of the mediating multiplication fact. Rather, the quotient generated may simply be associated with the presented division problem, increasing only the strength of the division fact in memory.

The present data demonstrated multiplication-todivision transfer, whereas Campbell (1997) did not observe multiplication-to-division error priming. This may 
reflect basic differences between the two phenomena. Transfer is facilitation measured between two corresponding (i.e., identical or closely related) problems (e.g., $6 \times$ $8,8 \times 6,48 \div 6$, or $48 \div 8$ ), whereas error priming is an interference effect measured between two different problems (e.g., $6 \times 8$ may prime the error $7 \times 6=48$; or $42 \div$ 7 may prime the error $8 \times 6=42$ ). With respect to multiplication-to-division transfer, a division probe would be an effective cue to retrieve the mediating multiplication fact, because corresponding division and multiplication problems share all their numerical components in common. With respect to error priming, in contrast, division problems presumably do not strongly activate noncorresponding multiplication nodes; consequently, noncorresponding nodes would be unlikely to become erroneously involved in mediation and to produce error priming. This may explain why we observe multiplicationto-division transfer but not multiplication-to-division error priming.

\section{Mediation Versus Checking of Division by Multiplication}

As was discussed in the preceding section, mediation might not produce division-to-multiplication transfer, but the multiplication check hypothesized by Campbell (1997) to account for division-to-multiplication error priming presumably would produce division-to-multiplication transfer, if checking occurred in the present study. In general, the division-to-multiplication error priming observed by Campbell (1997) is consistent with the view that people often access multiplication facts to either mediate or check their division performance, but the different asymmetries for short-lag transfer and error priming suggest either that transfer and error priming are governed by different factors (see the preceding section), or that using multiplication to check division was less likely in the present experiments. One good reason why checking would be less likely here is that the responsestimulus interval was only about $1-1.5 \mathrm{sec}$, as compared with $2.5 \mathrm{sec}$ in Campbell's (1997) study. Given that checking occurred between trials, the shorter response-stimulus interval in the present study would curtail checking.

More generally, however, when would people check division with multiplication, rather than mediate division by multiplication? The purpose of checking division with multiplication is, presumably, to detect errors. This implies that people know or assume that memory for multiplication is reliable, or at least more reliable than direct retrieval of division facts. In this case, however, we might expect people routinely to mediate division by multiplication, rather than only to check for an error after the division answer is produced. Nonetheless, checking (rather than mediation) would be expected to occur when performance demands or conditions of the task environment reduce opportunities for mediation but afford opportunities for checking. For example, conditions that emphasize speed over accuracy would reduce opportunities for mediation and promote increased reliance on direct re- trieval or guessing. The increased errors owing to speed pressure would also promote checking after the fact. Thus, speed pressure is likely both to reduce mediation and to increase checking. However, there must be adequate opportunity for checking to occur. Consequently, with very short intertrial intervals, as in the present experiment, or if intertrial intervals are filled with a distractor task, checking is likely to be curtailed.

\section{Implications for the Identical-Elements Model}

Methodological differences probably explain why Rickard and Bourne (1996; Rickard et al., 1994) found little or no transfer between division and multiplication in either direction, whereas the present study clearly demonstrated transfer from multiplication-to-division for large problems. First, we measured transfer over a range of only 4-6 trials here, whereas Rickard et al. tested transfer after longer delays (e.g., in separate test blocks at the end of practice or after a 1-day layoff from practice). Mediation may be promoted by recent retrieval of a potential mediator, favoring mediation in the present experiments more than in the Rickard et al. studies. Second, in the present studies, each problem in each operation was tested six times across blocks, and transfer tests were distributed across the 12 blocks. In contrast, Rickard et al. presented a large number of practice blocks ( 40 or 90 repetitions of practice items) prior to testing transfer to unpracticed items. It is possible that division performance was mediated over early practice blocks but was based on direct retrieval for the majority of practice trials. In this case, the effects of mediated transfer at test might have been weak, relative to those from associative transfer.

Despite the different methodologies and some differences in the pattern of transfer results, the present experiments confirmed an important prediction of the identical-elements model. Division-to-division transfer was larger with identical repetition than with an order change, whereas multiplication-to-multiplication transfer was equivalent for these two conditions. This operation $X$ order change interaction is predicted by the identicalelements model, according to which the two orders of division problems involve separate memory nodes, whereas the two orders of a multiplication problem access a common node. Associative transfer arises in the latter case, owing to strengthening of the common node. There was evidence of some transfer, however, between the two orders of division problems (e.g., $54 \div 6=9$ and $54 \div 9=$ 6). This is not predicted by the identical-elements model, but it does not necessarily contradict the model, if we distinguish between associative transfer (i.e., facilitation owing to strengthening of a common problem node) and mediated transfer (i.e., facilitation owing to mediation by a related problem). In the case of mediated transfer, anything that increases accessibility of the mediator (e.g., a recent practice trial) ought to yield facilitation for the mediated problem. Given independent nodes for the two orders of division problems, cross-order transfer in division would arise if both orders were mediated by a 
common, strengthened multiplication fact or if recent retrieval and strengthening of one order of a division problem was exploited to mediate performance of the other order. Greater savings with identical repetition than with an operand change in division might arise because more processing components are repeated in the case of identical repetition (i.e., encoding of stimulus information, retrieval processes, response production) and, therefore, more stages of processing may contribute savings.

Finally, the multiplication-to-division transfer observed here, although not predicted by the identical-elements model, is not inherently at odds with the model's representational assumptions. We found evidence that large division problems were mediated by multiplication, but there seems to be no reason in principle why people cannot develop memory processes for division facts that do not involve multiplication. Indeed, the absence of transfer from small multiplication to small division problems supports this and is consistent with the identicalelements model. Nonetheless, the present data suggest that skilled adults apparently do utilize multiplication knowledge in their processing of large-number division facts, at least under conditions in which potential mediators are readily accessible.

\section{CONCLUDING COMMENTS}

Recall that Hittmair-Delazer et al. (1994) described an acalculic patient whose division performance apparently depended on multiplication facts, whereas Cipolotti and de Lacy Costello (1995) studied an acalculic patient with largely intact multiplication performance but severely impaired division. Mediation of division by multiplication undoubtedly depends on a variety of factors, and individual differences in these factors may explain the apparently inconsistent neuropsychological evidence. Individuals with very well developed memory for division facts presumably rely very little on mediation. Similarly, individuals who have poorly developed memory for multiplication are unlikely to rely on multiplication to perform division. It would not be surprising for mediation to be common, however. LeFevre, Bisanz, et al. (1996; LeFevre, Sadesky, \& Bisanz, 1996) concluded that well-educated adults often use a variety of nonretrieval strategies to perform simple arithmetic problems, especially largenumber problems, which typically are not as well memorized. The present results suggest similarly that mediation of division by multiplication may be a persistent strategy or process used by skilled adults. Although simple direct retrieval is probably the modal process used by educated adults for basic arithmetic, the emerging evidence suggests that adults also possess a repertoire of efficient, conceptually based procedures for simple arithmetic that researchers are only beginning to recognize and study.

\section{REFERENCES}

ASHCRAFT, M. H. (1992). Cognitive arithmetic: A review of theory and data. Cognition, 44, 75-106.
AshCRAFT, M. H. (1995). Cognitive psychology and simple arithmetic: A review and summary of new directions. Mathematical Cognition, 1,3-34.

AshCRAFT, M. H., \& Christy, K. S. (1995). The frequency of arithmetic facts in elementary texts: Addition and multiplication in grades 1-6. Journal for Research in Mathematics Education, 5, 396-421.

BAROODY, A. J. (1994). An evaluation of evidence supporting factretrieval models. Learning \& Individual Differences, 6, 1-36.

CAmpBell, J. I. D. (1995). Mechanisms of simple addition and multiplication: A modified network-interference theory and simulation. Mathematical Cognition, 1, 121-164.

CAMPBELL, J. I. D. (1997). On the relation between skilled performance of simple division and multiplication. Journal of Experimental Psychology: Learning, Memory, \& Cognition, 23, 1140-1159.

Camprell, J. I. D., \& Clark, J. M. (1989). Time course of error-priming in number fact retrieval: Evidence for excitatory and inhibitory mechanisms. Journal of Experimental Psychology: Learning, Memory, \& Cognition, 15, 920-929.

Cipolottr, L., \& de Lacy Costello, A. (1995). Selective impairment for simple division. Cortex, 31, 433-449.

GEARY, D. C. (1994). Children's mathematical development. Washington, DC: American Psychological Association.

GEARY, D. C. (1996). The problem size effect in mental addition: Developmental and cross-national trends. Mathematical Cognition, 2, 63-93.

Geary, D. C., \& Wiley, J. G. (1991). Cognitive addition: Strategy choice and speed-of-processing differences in young and elderly adults. Psychology \& Aging, 6, 474-483.

Hittmair-Delazer, M., Semenza, C., \& Denes, G. (1994). Concepts and facts in calculation. Brain, 117, 715-728.

LeFevre, J., Bisanz, J., Daley, K. E., Buffone, L., Greenham, S. L., \& SADESKY, G. S. (1996). Multiple routes to solution of single-digit multiplication problems. Journal of Experimental Psychology: General, 125, 284-306.

LeFevre, J., SAdesky, G. S., \& Bisanz, J. (1996). Selection of procedures in mental addition: Reassessing the problem size effect in adults. Journal of Experimental Psychology: Learning, Memory, \& Cognition, 22, 216-230.

RickARD, T. C., \& BourNE, L. E., JR. (1996). Some tests of an identical elements model of basic arithmetic skills. Journal of Experimental Psychology: Learning, Memory, \& Cognition, 22, 1281-1295.

Rickard, T. C., Healy, A. F., \& Bourne, L. E., JR. (1994). On the representation of arithmetic facts: Operand order, symbol, and operation transfer effects. Journal of Experimental Psychology: Learning, Memory, \& Cognition, 20, 1139-1153.

SIEGLER, R. S. (1988). Strategy choice procedures and the development of multiplication skill. Journal of Experimental Psychology: General, 117, 258-275.

ZBRODOFF, N. J. (1995). Why is $9+7$ harder than $2+3$ ? Strength and interference as explanations for the problem-size effect. Memory \& Cognition, 23, 689-700.

\section{NOTE}

1. Among the 14 small problems, half involve repeated products that are correct for two combinations of operands (i.e., 12, 16, 18, or 24), whereas there is only one repeated product (i.e., 36) among the large problems. Because they are not associated with a unique problem, items with repeated product/dividends might produce weak transfer. In this case, the high proportion of small problems with repeated product/dividends could contribute to the finding that small-problem multiplication primes did not produce transfer to division. To check this, a four-factor ANOVA (operation, transfer condition, lag, and repeated vs. unique products/dividends) of the combined data that included only smallnumber problems was performed separately for RT and errors. There was no evidence of differential transfer for items with unique versus repeated products/dividends, either for RT or for errors.
(Manuscript received December 18, 1997; revision accepted for publication October 5, 1998.) 